\title{
I 24 Delayed-enhancement MRI as a predictor of functional recovery after revascularization: results from an International Multicenter Viability Trial
}

\author{
Veronica V Lenge*1, Raja Muthupillai ${ }^{2}$, Harrie Van den Bosch ${ }^{3}$, \\ John Greenwood ${ }^{4}$, Rungroj Krittyaphong5, Wong Yong Kim6, Reza Razavi , \\ Peter Danias ${ }^{8}$, Eike Nagel ${ }^{9}$ and Scott D Flamm ${ }^{10}$
}

\begin{abstract}
Address: ${ }^{1}$ Baylor College of Medicine, Houston, TX, USA, ${ }^{2}$ St Luke's Episcopal Hospital - THI, Houston, TX, USA, ${ }^{3}$ Catharina Ziekenhuis Hospital, Eindhoven, The Netherlands, ${ }^{4}$ Leeds General Infirmary, Leeds, UK, ${ }^{5}$ Siriraj Hospital, Bangkok, Thailand, ${ }^{6}$ Skejby University Hospital, Aarhus, Denmark, ${ }^{7}$ Guy's Hospital, London, UK, ${ }^{8}$ Hygeia Hospital, Maroussi, Greece, ${ }^{9}$ German Heart Institute Berlin, Berlin, Germany and ${ }^{10}$ Cleveland Clinic Foundation, Cleveland, OH, USA

* Corresponding author
\end{abstract}

from I th Annual SCMR Scientific Sessions

Los Angeles, CA, USA. I-3 February 2008

Published: 22 October 2008

Journal of Cardiovascular Magnetic Resonance 2008, I0(SuppI I):A25 doi:I0.I I86/I532-429X-10-SI-A25

This abstract is available from: http://jcmr-online.com/content/I0/SI/A25

(c) 2008 Lenge et al; licensee BioMed Central Ltd.

\section{Purpose}

Several single center studies have shown that Delayedenhancement (MRI DE-MRI) can predict functional recovery after revascularization $[1,2]$. The purpose of this study was to determine the prognostic value of DE-MRI for predicting recovery of regional wall motion abnormality after revascularization in patients with chronic coronary artery disease (CAD) in a prospective, international, multicenter trial.

\section{Methods}

183 patients ( 167 men, age: $59.1 \pm 9.7$ years) with angiographically confirmed CAD and resting wall motion abnormality (WMA) scheduled for coronary bypass $(\mathrm{n}=$ $101)$ or angioplasty $(n=82)$ were enrolled from 9 institutions (US, Europe, and Asia) using a standardized imaging protocol. Functional and DE-MRI were performed within 30 days prior to and $5.6 \pm 2.4$ months after intervention.

\section{Data Acquisition}

All images were acquired on a Philips $1.5 \mathrm{~T}$ imager with VCG gating, and a five-element synergy cardiac coil was used for signal reception. A steady-state free precession (SSFP) sequence was used for cardiac cine imaging: TR/ TE/flip: $2.7 \mathrm{msec} / 1.3 \mathrm{msec} / 65^{\circ}$; FOV: $320 \mathrm{~mm}$, matrix:
$160 \times 160$, slice thickness: $10 \mathrm{~mm}$; slice gap: $0 \mathrm{~mm}$; acquired voxel size: $2.0 \times 2.0 \times 10.0 \mathrm{~mm}$ and reconstructed voxel size: $1.3 \times 1.3 \times 10.0 \mathrm{~mm}$; temporal resolution: $25-40$ msec depending on heart rate. Cine SSFP images were acquired in the two long - axis orientations (vertical long axis and 4 chambers) and short axis orientations (10 to 12 contiguous short-axis cine SSFP images from mitral valve plane through the apex). DE-MRI images were acquired 15 minutes after administering 0.2 $\mathrm{mmol} / \mathrm{kg}$ of gadolinium-chelate (OptiMARK ${ }^{\mathrm{TM}}$ ) in the same orientation as the cine images. The DE-MRI sequence was an inversion-recovery prepared, segmented gradient echo acquisition with the following acquisition parameters: field-of-view: $384 \times 384 \mathrm{~mm}$; matrix size: 256 $\times 256$; acquired voxel size: $1.5 \times 1.5 \times 10 \mathrm{~mm}$; 32 phase encoding steps per RR interval; TR/TE/flip: $4.6 / 1.9 / 15^{\circ}$; NSA: 2; acquisition time per slice: 16 RR intervals. The inversion delay time (TI) between the inversion pulse and the beginning of the data acquisition necessary to null the signal from normal myocardium was determined by the Look-Locker sequence. The data acquisition was timed to occur in diastole to minimize cardiac motion. 


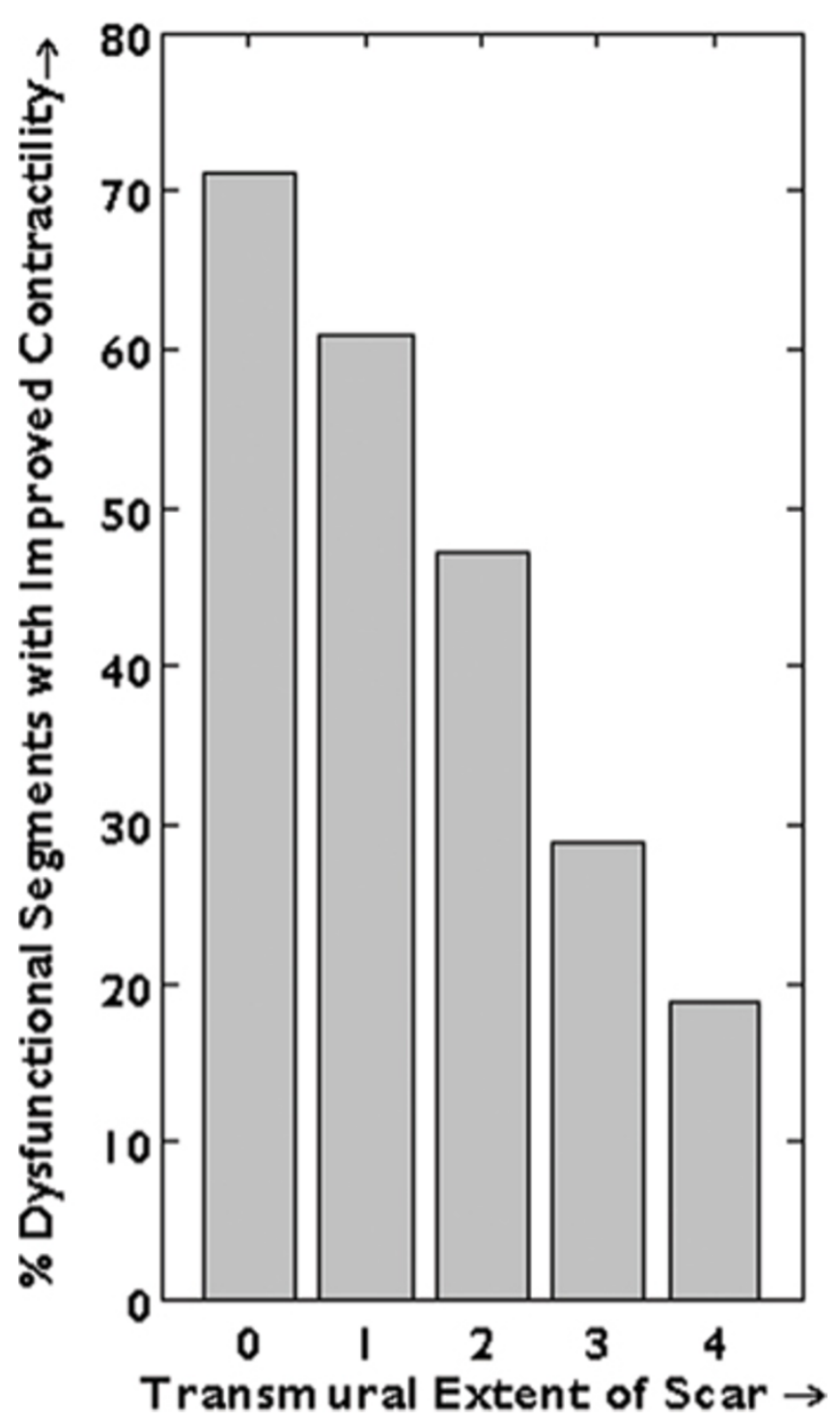

Figure I

The Multicenter Viability Trial confirmed the prognostic value of DE-MRI for predicting recovery of left ventricular function recovery after revascularization in patients with chronic coronary artery disease (CAD).

\section{Data Analysis}

Cine and delayed - enhanced images were analyzed based on the model proposed by Kim R et al [1] in which the left ventricle was divided into 12 equiangular segments on 8 - 10 short - axis views, depending on the length of ventricle. Progressively worsening segmental WMA and increasing extent of DE were scored on a scale of $0-4$ [1].

\section{Results}

At baseline, 5580/12274 (46\%) segments had abnormal contractility, and 2974/12274 (24\%) segments had DE. Improvement in contractility of impaired segments fol- lowing revascularization correlated inversely with the transmural extent of scar $\left(\mathrm{r}^{2}=0.9\right.$, Fig. 1). For instance, $72 \%$ of dysfunctional segments without evidence of scar at baseline showed improvement in contractility following revascularization, and remarkably, 52\% of such segments had complete recovery of function ( $\mathrm{p}<0.001)$. Conversely, less than a fourth $(250 / 1438,17 \%)$ of the segments with near or complete transmural scar (score 3 and 4 ) had any functional recovery ( $P$ value $<0.001)$.

\section{Conclusion}

The results from the first, global, multi-center trial determined that DE-MRI is a robust predictor of functional recovery after revascularization.

\section{References}

I. Kim RJ, et al.: The use of contrast-enhanced magnetic resonance imaging to identify reversible myocardial dysfunction. N Engl J Med 2000, 343: I 445-53.

2. Selvanayagam JB, et al:: Value of delayed-enhancement cardiovascular magnetic resonance imaging in predicting myocardial viability after surgical revascularization. Circulation 2004, I I 0:1535-4I. 